PROCEEDINGS OF THE

AMERICAN MATHEMATICAL SOCIETY

Volume 129, Number 4 , Pages 1161-1168

S 0002-9939(00)05587-8

Article electronically published on September 20, 2000

\title{
CONSTRUCTING THE KÄHLER AND THE SYMPLECTIC STRUCTURES FROM CERTAIN SPINORS ON 4-MANIFOLDS
}

\author{
Y. BYUN, Y. LEE, J. PARK, AND J. S. RYU \\ (Communicated by Ronald A. Fintushel)
}

\begin{abstract}
We show that, on an oriented Riemannian 4-manifold, existence of a non-zero parallel spinor with respect to a $\operatorname{spin}^{c}$ structure implies that the underlying smooth manifold admits a Kähler structure. A similar but weaker condition is obtained for the 4-manifold to admit a symplectic structure. We also show that the $\operatorname{spin}^{c}$ structure in which the non-zero parallel spinor lives is equivalent to the canonical $\operatorname{spin}^{c}$ structure associated to the Kähler structure.
\end{abstract}

\section{INTRODUCTION}

Let $X$ be a Kähler manifold and consider the canonical spin ${ }^{c}$ structure. Then the spin bundle can be identified with the exterior algebra of the complex antilinear cotangent bundle, $\bigwedge^{0, *}(X, \mathbb{C})$ (cf. $\left.\S 3.4,[2]\right)$. Furthermore, the Levi-Civita connection on $\bigwedge^{0, *}(X, \mathbb{C})$ corresponds to a spin ${ }^{c}$ connection and it follows that a constant function is a parallel spinor. Thus a Kähler manifold admits a non-zero parallel spinor. If $X$ is only symplectic, which is somewhat weaker than being Kähler, still the spin bundle can be identified with $\bigwedge^{0, *}(X, \mathbb{C})$ assuming a choice of an almost complex structure on the tangent bundle $T X$. Then for an appropriate choice of $\operatorname{spin}^{c}$ connection, the constant function is a harmonic spinor ([6]). The main purpose of this paper is to show that the converses for these two observations hold for 4-manifolds.

Note that any closed oriented smooth Riemannian 4-manifold admits a spin ${ }^{c}$ structure, even if we will not demand the closedness condition for the manifolds.

Theorem 1. Assume an oriented Riemannian 4-manifold $X$ admits a spin ${ }^{c}$ structure with a non-zero spinor which is parallel with respect to a spin ${ }^{c}$ connection. Then the smooth manifold $X$ admits a Kähler structure.

In the above we allow the orientation of the Kähler structure to be opposite to the original one, which applies to Theorem 2 below as well.

Received by the editors April 30, 1999 and, in revised form, June 21, 1999.

1991 Mathematics Subject Classification. Primary 53C05, 53C07.

Key words and phrases. Parallel positive spinor, Kähler manifold, symplectic manifold, spin ${ }^{c}$ structure.

The first author was partially supported by the Hanyang University Research Fund. The second author was supported in part by 1998-015-D00044. The third author was partially supported by the Dongguk University Research Fund. The fourth author was supported in part by GARC. 
Theorem 2. Assume an oriented Riemannian 4-manifold $X$ has a spin ${ }^{c}$ structure for which there is a non-zero positive harmonic spinor $\psi$ such that

$$
\left\langle\tilde{\nabla}_{v} \psi, \psi\right\rangle=0
$$

for any $v \in T X$, where $\langle\cdot, \cdot\rangle$ denotes the Hermitian metric for the spin bundle and $\tilde{\nabla}$ is the spin ${ }^{c}$ connection. Then the smooth manifold $X$ admits a symplectic structure.

Here that $\psi$ is harmonic means that it is in the kernel of a Dirac operator. The main ideas for both the statement and the proof of Theorem 2 are due to C. H. Taubes [6].

It is interesting to compare Theorem 1 1 with a statement by A. Moroianu [3]: A

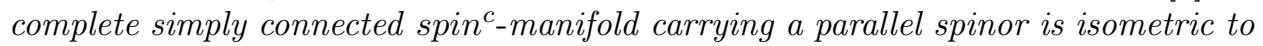
the Riemannian product between a Kähler manifold and a spin manifold carrying a parallel spinor. On the other hand it is shown by M. Wang (7] and also see [1) that a complete, simply-connected irreducible spin 4-manifold having a non-zero spin field is Kähler. Therefore, their results combined imply that a simply-connected complete 4-manifold carrying a parallel spinor is Kähler. However the simplyconnectedness condition and the completeness one are essential for their arguments which exploit such theorems as the de Rham decomposition theorem. Our paper assumes neither completeness nor simply-connectedness for the manifolds.

Note that in each of Theorem 1 and 2, two spin ${ }^{c}$ structures, one of them being implicit, are involved. In fact, we will see that, given a nowhere vanishing positive spinor $\psi$ in a $\operatorname{spin}^{c}$ structure on a 4 -manifold $X$, it determines a 2 -form $\omega$ which is nondegenerate at every point of $X$. In turn $\omega$ gives rise to a $\operatorname{spin}^{c}$ structure well-defined up to an equivalence, which we will refer to as the $\operatorname{spin}^{c}$ structure determined by $\psi$. The details of this construction are provided in $₫ 4$ below Lemma 4.1 .

For the special case when $\psi$ is parallel, we have:

Theorem 3. Let $X$ be an oriented Riemannian 4-manifold which admits a spin ${ }^{c}$ structure. Assume there is a non-zero positive spinor $\psi$ which is parallel with respect to a spin ${ }^{c}$ connection. Then the spin ${ }^{c}$ structure determined by $\psi$ is equivalent to the original one.

At the moment we do not know whether the above holds for general nowhere vanishing positive spinors.

\section{Preliminaries}

Let $(X, g)$ be an oriented Riemannian 4-manifold, which is not necessarily compact and $P$ denote the oriented tangent frame bundle of $X$. A spin ${ }^{c}$ structure on $X$ is a smooth map $\tilde{P} \rightarrow P$, in which $\tilde{P}$ is a principal $\operatorname{Spin}^{c}(4)$ bundle over $X$ and the map respects the actions of $\operatorname{Spin}^{c}(4), S O(4)$ by means of the homomorphism $\operatorname{Spin}^{c}(4) \rightarrow S O(4)$. Fix an irreducible complex representation of the complexified Clifford algebra, $\rho: C l(4) \otimes \mathbb{C} \rightarrow E n d_{\mathbb{C}} S_{\mathbb{C}}(4)$. We also choose a Hermitian metric on $S_{\mathbb{C}}(4)$ so that $\rho$ maps $\operatorname{Spin}^{c}(4)$ into the group of unitary automorphisms of $S_{\mathbb{C}}(4)$. Then we obtain a Hermitian complex vector bundle $S_{\mathbb{C}}(\tilde{P})=\tilde{P} \times{ }_{\rho} S_{\mathbb{C}}(4)$, which is a $C l(T X) \otimes \mathbb{C}$ module. $S_{\mathbb{C}}(\tilde{P})$ is commonly referred to as the spin bundle on $X$ associated to the $\operatorname{spin}^{c}$ structure $\tilde{P} \rightarrow P$. 
Let $\nabla$ denote the Levi-Civita connection on $T X$ and let $A$ be a connection on the determinant line bundle $\mathcal{L}=\tilde{P} \times_{\operatorname{Spin}^{c}(4)} \mathbb{C}$, in which $\operatorname{Spin}^{c}(4)$ acts on $\mathbb{C}$ via the homomorphism $\operatorname{Spin}^{c}(4) \rightarrow S^{1}$. Then there is a unique connection $\nabla^{A}$ on $S_{\mathbb{C}}(\tilde{P})$ determined by both $\nabla$ and $A$, which is a $\operatorname{spin}^{c}$ connection (cf. [4], [6]) in the sense that the Hermitian metric on $S_{\mathbb{C}}(\tilde{P})$ is parallel and the following form of Leibniz rule holds:

$$
\nabla_{v}^{A}(\omega \cdot \psi)=\left(\nabla_{v} \omega\right) \cdot \psi+\omega \cdot \nabla_{v}^{A} \psi
$$

where $v$ is a tangent vector of $X, \omega$ is a complex valued $k$-form on $X$ and $\psi$ is a smooth section of $S_{\mathbb{C}}(\tilde{P})$, that is, a spinor. Here '.' denotes the Clifford multiplication of a spin vector by a complex valued $k$-form, which is defined by a natural complex bundle isomorphism, $\bigwedge^{*}\left(T^{*} X\right) \otimes \mathbb{C} \rightarrow C l\left(T^{*} X\right) \otimes \mathbb{C}$, followed by the isomorphism $C l\left(T^{*} X\right) \otimes \mathbb{C} \rightarrow C l(T X) \otimes \mathbb{C}$ determined by the Riemannian metric $g$. In fact every $\operatorname{spin}^{c}$ connection is $\nabla^{A}$ for some $A$ (cf. [4).

Note that the volume element of the Clifford algebra $C l(T X) \otimes \mathbb{C}$ corresponds to $-\Omega$, where $\Omega$ denotes the volume form of $(X, g)$. Then $S_{\mathbb{C}}(\tilde{P})$ can be decomposed into $S_{\mathbb{C}}^{+}(\tilde{P})$ and $S_{\mathbb{C}}^{-}(\tilde{P})$, which respectively consist of \pm 1 eigenspaces of the volume element.

Now let $\Omega\left(S_{\mathbb{C}}(\tilde{P})\right)$ and $\Omega\left(S_{\mathbb{C}}^{+}(\tilde{P})\right), \Omega\left(S_{\mathbb{C}}^{-}(\tilde{P})\right)$ denote the sets of smooth sections. Then the Dirac operator, $D^{A}: \Omega\left(S_{\mathbb{C}}(\tilde{P})\right) \rightarrow \Omega\left(S_{\mathbb{C}}(\tilde{P})\right)$, is defined by

$$
\left(D^{A} \psi\right)_{x}=\sum_{i=1}^{4} e^{i} \cdot \nabla_{e_{i}}^{A} \psi
$$

for any $\psi \in \Omega\left(S_{\mathbb{C}}(\tilde{P})\right)$ and any $x \in X$, where $e_{i}, i=1,2,3,4$, denotes an orthonormal basis of $T_{x} X$ and $e^{i}, i=1,2,3,4$, its dual. Note that the Dirac operator restricts to

$$
D^{A}: \Omega\left(S_{\mathbb{C}}^{ \pm}(\tilde{P})\right) \rightarrow \Omega\left(S_{\mathbb{C}}^{\mp}(\tilde{P})\right) .
$$

Finally, a symplectic structure on $X$ is given by a closed 2 -form $\omega$ such that $\omega_{x}: T_{x} X \times T_{x} X \rightarrow \mathbb{R}$ is non-degenerate for each $x \in X$. A Kähler structure on $X$ consists of a symplectic 2-form $\omega \in \Omega^{2}(X, \mathbb{R})$ together with an almost complex structure $J$ so that the expression $g_{\omega, J}(v, w)=\omega(v, J w), v, w \in T_{x} X$, defines a Riemannian metric and $J$ is parallel with respect to the Levi-Civita connection associated to $g_{\omega, J}$.

\section{The Kähler AND THE SYMPLECTIC STRUCTURES}

In this section we prove Theorems 1 and 2

We start from the observation that a positive spinor determines a self-dual realvalued 2-form: Let $\psi \in \Omega\left(S_{\mathbb{C}}^{+}(\tilde{P})\right)$ where $\tilde{P}$ is a $\operatorname{spin}^{c}$ structure on an oriented Riemannian 4-manifold $X$. Then we consider the endomorphism of $S_{\mathbb{C}}^{+}(\tilde{P})$ given by

$$
q(\psi)=\psi \otimes \psi^{*}-\frac{1}{2}|\psi|^{2} I d .
$$

Here we exploit the fact that the traceless self-adjoint endomorphisms of $S_{\mathbb{C}}^{+}(\tilde{P})$ are in 1-1 correspondence with the self-dual purely imaginary 2-forms on $X$ where the 1-1 correspondence is given by the Clifford multiplication (cf. pp.55-57, [2]). It is straightforward to see that $q(\psi)$ is traceless and self-adjoint. Thus there exists a real-valued self-dual 2 -form $\omega$ corresponding to $-i q(\psi)$. 
Terminology. We will say that $\omega$ in the above is determined by $\psi$.

Lemma 3.1. Let $\psi$ be a positive spinor on an oriented Riemannian 4-manifold $X$, presuming a spin ${ }^{c}$ structure on $X$, and let $\omega$ be the self-dual real-valued 2form determined by $\psi$. Then, for any $x \in X$ such that $\psi_{x} \neq 0$, we have that $\omega_{x}: T_{x} X \times T_{x} X \rightarrow \mathbb{R}$ is nondegenerate.

Proof. It is enough to note that $\omega \wedge \omega=\omega \wedge(* \omega)=|\omega|^{2} \Omega$, where $\Omega$ denotes the volume form of $X$, and that $\omega_{x}$ is non-zero if $\psi_{x}$ is non-zero.

Proof of Theorem 1. Let $\psi$ be a non-zero section in a spin bundle $S_{\mathbb{C}}(\tilde{P})$ on $X$ which is parallel with respect to a $\operatorname{spin}^{c}$ connection $\nabla^{A}$.

Then $\psi^{+}$, the $S_{\mathbb{C}}^{+}(\tilde{P})$-component of $\psi$, is a non-zero parallel spinor, reversing the orientation if necessary. Thus without loss of generality we may assume $\psi$ itself is a non-zero positive parallel spin field.

The proof is divided into two steps.

The Kähler form: Let $\omega$ denote the self-dual real-valued 2-form determined by $\psi$.

Since the Hermitian metric on $S_{\mathbb{C}}(\tilde{P})$ is parallel with respect to $\nabla^{A}$, it follows that $|\psi|$ is constant: we have $d\langle\psi, \psi\rangle=\left\langle\nabla^{A} \psi, \psi\right\rangle+\left\langle\psi, \nabla^{A} \psi\right\rangle=0$. In particular, it follows that $\omega$ is non-degenerate at every point of $X$ by Lemma 3.1.

Note that $\omega \cdot \psi=-\frac{i}{2}|\psi|^{2} \psi=-i k \psi$, writing $k$ for the constant $\frac{1}{2}|\psi|^{2}$. Therefore, we have

$$
0=\nabla_{v}^{A}(\omega \cdot \psi)=\left(\nabla_{v} \omega\right) \cdot \psi_{x}
$$

where $\nabla$ denotes the Levi-Civita connection and $v$ is a tangent vector based at $x \in X$. On the other hand, $\nabla$ preserves the self-dual 2 -forms. Thus $\nabla_{v} \omega$ acts as a traceless skew adjoint endomorphism of $S_{\mathbb{C}}^{+}(\tilde{P})_{x}$ by the Clifford multiplication. Since it has turned out that one of its eigenvalues is zero, the other eigenvalue must be zero as well. Thus we have: $\nabla \omega=0$.

The almost complex structure: Let $A: T X \rightarrow T X$ be the endomorphism defined by $g(v, A w)=\omega(v, w)$. Then $A$ is a skew adjoint automorphism of $T X$. Furthermore, $A$ is parallel with respect to the Levi-Civita connection $\nabla$ associated to $g$ since $g$ and $\omega$ are parallel.

Here we recall the following fact: Let $E \rightarrow X$ be a smooth real vector bundle of finite rank with a Riemannian metric $h$ and let $S: E \rightarrow E$ be a smooth bundle isomorphism which is self-adjoint and positive definite. Then there is a unique self-adjoint positive definite smooth bundle isomorphism $\sqrt{S}: E \rightarrow E$ satisfying $\sqrt{S}^{2}=S$. Furthermore, for any connection $\nabla^{\prime}$ on $E$ such that $\nabla^{\prime} S=0$, it holds that $\nabla^{\prime} \sqrt{S}=0$ since, by applying the covariant derivative to the both sides of the equality, $\sqrt{S}^{2}=S$, we have

$$
\left(\nabla_{v}^{\prime} \sqrt{S}\right) \sqrt{S}_{x}+\sqrt{S}_{x}\left(\nabla_{v}^{\prime} \sqrt{S}\right)=0
$$

for any tangent vector $v$ of $X$ based at $x \in X$. By applying both sides of this equation to each of the eigenvectors of $\sqrt{S}$ we conclude that $\nabla_{v}^{\prime} \sqrt{S}=0$.

Now we set $J=-A{\sqrt{-A^{2}}}^{-1}$ as the almost complex structure. First of all, we have $J^{2}=-I d$. Also writing $\hat{A}=\sqrt{-A^{2}}$, we have

$$
\omega(v, J w)=g(v, \hat{A} w) .
$$


Since $\hat{A}$ is a self-adjoint positive definite endomorphism, $J$ is compatible with $\omega$. Furthermore, since $\nabla \hat{A}=0$, the Levi-Civita connection associated to $(\omega, J)$ is exactly $\nabla$ itself. Finally, since $\nabla J=0$, we conclude that $(\omega, J)$ defines a Kähler structure on $X$.

Proof of Theorem 2. Again we consider the self-dual real-valued 2-form $\omega$ determined by $\psi$. Then the argument of the proof of Theorem 1 is still valid to show that $\omega$ is nondegenerate since still we have $d\langle\psi, \psi\rangle=\left\langle\nabla^{A} \psi, \psi\right\rangle+\left\langle\psi, \nabla^{A} \psi\right\rangle=0$.

It remains to show that

$$
d \omega=0 .
$$

By definition we have $\omega \cdot \psi=-i k \psi$, where $k$ is the constant $\frac{1}{2}|\psi|^{2}$. Apply the Dirac operator $D^{A}$ to the both sides of this equality to obtain the equality:

$$
\sum_{i=1}^{4} e^{i} \cdot\left(\nabla_{e_{i}} \omega \cdot \psi+\omega \cdot \nabla_{e_{i}}^{A} \psi\right)=0,
$$

where $e_{i}, i=1,2,3,4$, is an orthonormal basis of $T_{x} X$ and $e^{i}, i=1,2,3,4$, is its dual.

Note that we have

$$
\sum_{i=1}^{4} e^{i} \cdot \nabla_{e_{i}} \omega=\sum_{i=1}^{4}\left(e^{i} \wedge\left(\nabla_{e_{i}} \omega\right)-e_{i} \angle\left(\nabla_{e_{i}} \omega\right)\right)=d \omega-d^{*} \omega=(1-*) d \omega .
$$

Furthermore, we have $* \theta=(-\Omega) \cdot \theta$ and for any 3 -form $\theta$ where $\Omega$ is the volume form of $X$. Note that $-\Omega$ corresponds to the volume element of the Clifford algebra bundle of $T^{*} X$. Since $d \omega \cdot \psi$ is a section of $S_{\mathbb{C}}^{-}(\tilde{P})$, we conclude that

$$
2(d \omega) \cdot \psi=-\sum_{i=1}^{4} e^{i} \cdot\left(\omega \cdot \nabla_{e_{i}}^{A} \psi\right)
$$

Now by definition we have

$$
\omega \cdot \nabla_{e_{i}}^{A} \psi=-i q(\psi)\left(\nabla_{e_{i}}^{A} \psi\right)=i k \nabla_{e_{i}}^{A} \psi
$$

since by assumption $\left\langle\nabla_{v}^{A} \psi, \psi\right\rangle=0$ for any $v \in T X$. Thus we have

$$
\sum_{i=1}^{4} e^{i} \cdot\left(\omega \cdot \nabla_{e_{i}}^{A} \psi\right)=i k D^{A} \psi=0
$$

Therefore we conclude that

$$
2(d \omega) \cdot \psi=0 .
$$

Since a 1 -form $\theta, \theta_{x} \neq 0, x \in X$, acts on the fiber $S_{\mathbb{C}}(\tilde{P})_{x}$ as an isomorphism by the Clifford multiplication, it follows that the same is true for a 3 -form since $X$ is a four manifold. Thus we conclude that $d \omega=0$.

\section{THE $\operatorname{SPIN}^{c}$ STRUCTURES}

Let $\tilde{P}_{g} \rightarrow P_{g}, \tilde{P}_{g^{\prime}} \rightarrow P_{g^{\prime}}$ be two spin $^{c}$ structures on an oriented $n$-manifold $X$, where $g, g^{\prime}$ are Riemannian metrics on $X$ and $P_{g}, P_{g^{\prime}}$ denote the respective principal $S O(n)$ bundles consisting of the oriented orthonormal frames.

Then it is well known that $\tilde{P}_{g}, \tilde{P}_{g^{\prime}}$ are isomorphic to each other as principal $\operatorname{Spin}^{c}(n)$ bundles over $X$ if and only if their respective determinant $S^{1}$ bundles are isomorphic to each other, which are given by the homomorphism, det : $\operatorname{Spin}^{c}(n) \rightarrow$ 
$S^{1}$. Also it is well known that the complex line bundle associated to the determinant $S^{1}$ bundle of a $\operatorname{Spin}^{c}(n)$ bundle is isomorphic to the determinant line bundle of the positive spin bundle. Therefore we have:

Lemma 4.1. Let $X$ be a smooth oriented n-manifold, $g, g^{\prime}$, two Riemannian metrics on $X$ and $P_{g}, P_{g^{\prime}}$, the respective oriented tangent frame bundles. Assume there are spin ${ }^{c}$ structures $\tilde{P}_{g} \rightarrow P_{g}, \tilde{P}_{g^{\prime}} \rightarrow P_{g^{\prime}}$. Then the two spin ${ }^{c}$ structures are equivalent to each other if and only if $S_{\mathbb{C}}^{+}\left(\tilde{P}_{g}\right), S_{\mathbb{C}}^{+}\left(\tilde{P}_{g^{\prime}}\right)$ are isomorphic to each other as complex vector bundles.

In the above by writing that two $\operatorname{spin}^{c}$ structures are equivalent to each other we mean the two principal $\operatorname{Spin}^{c}(n)$ bundles, $\tilde{P}_{g}, \tilde{P}_{g^{\prime}}$, are isomorphic to each other.

Now let $(X, g)$ be an oriented Riemannian 4-manifold which admits a $\operatorname{spin}^{c}$ structure, $\tilde{P}_{g} \rightarrow P_{g}$, and assume there is a nowhere vanishing positive spinor $\psi$ with respect to the $\operatorname{spin}^{c}$ structure. Then the 2 -form $\omega$ on $X$ determined by $\psi$ is nondegenerate at each point of $X$ by Lemma 3.1 Let $J$ denote an almost complex structure on $X$ which is compatible with $\omega$ in the sense the expression $h(v, w)=$ $\omega(v, J w)$ defines a Riemannian metric $h$ on $X$. Such almost complex structures always exist and they form a contractible space (cf. Proposition 4.1, [5]). Then $J$ determines a $\operatorname{spin}^{c}$ structure, say, $\tilde{P}_{\omega, J} \rightarrow P_{\omega, J}$ on $(X, h)$ whose spin bundle can be identified with $\bigwedge^{0, *}(X, \mathbb{C})$. Note that the $\operatorname{spin}^{c}$ structure, $\tilde{P}_{\omega, J} \rightarrow P_{\omega, J}$, does not depend on the choice of $J$ up to equivalence.

Now we may restate Theorem 3 .

Theorem 4. Assume $\psi$ is parallel. Then the two spin ${ }^{c}$ structures, $\tilde{P}_{g} \rightarrow P_{g}$, $\tilde{P}_{\omega, J} \rightarrow P_{\omega, J}$ are equivalent to each other.

Proof. The proof is divided into two cases.

Case 1: The vector space of parallel positive spinors is of rank 1. Note that $\bigwedge^{0, \text { even }}(X, \mathbb{C})$ is isomorphic to $S_{\mathbb{C}}^{+}\left(\tilde{P}_{\omega, J}\right)$ as a $\left(C l_{h, 0}\left(T^{*} X\right) \otimes \mathbb{C}\right)^{+}$module. On the other hand, $\bigwedge^{0,2}(X, \mathbb{C})$ can be also viewed as a subbundle of $\left(C l_{h, 0}\left(T^{*} X\right) \otimes \mathbb{C}\right)^{+}$ by the canonical identification of $\Lambda^{*}(X, \mathbb{C})$ with $C l_{h}\left(T^{*} X\right) \otimes \mathbb{C}$.

Let $h$ denote the Riemannian metric on $X$ determined by $\omega, J$. Then first consider the endomorphism $A: T X \rightarrow T X$ defined by $h(v, w)=g(v, A w)$ and subsequently set $S: T X \rightarrow T X$ as the 'square-root' of $A$ (see the proof Theorem (1). Note that $S$ satisfies $h(v, w)=g(S v, S w)$ for any $v, w \in T_{x} X, x \in X$ and also that $S$ is self-adjoint with respect to both $g, h$, positive definite and parallel with respect to the Levi-Civita connection associated $g, h$.

Let $S: C l_{h}\left(T^{*} X\right) \otimes C \rightarrow C l_{g}\left(T^{*} X\right) \otimes C$ denote the isomorphism induced by $S: T X \rightarrow T X$, allowing ourselves a slight abuse of notation. Then $S$ maps $\left(C l_{h, 0}(X) \otimes \mathbb{C}\right)^{+}$isomorphically into $\left(C l_{g, 0}\left(T^{*} X\right) \otimes \mathbb{C}\right)^{+}$. Therefore, we have a well defined bundle homomorphism:

$$
S_{\mathbb{C}}^{+}\left(\tilde{P}_{\omega, J}\right) \equiv \bigwedge^{0, \text { even }}(X, \mathbb{C}) \rightarrow S_{\mathbb{C}}^{+}\left(\tilde{P}_{g}\right), a \rightarrow S(a) \cdot \psi .
$$

Considering Lemma 4.1, the following is enough to show that this is an isomorphism between complex vector bundles.

Claim. If $a \in \bigwedge^{0,2}(X, \mathbb{C}), a \neq 0$, then $S(a) \cdot \psi$ and $\psi=S(1) \cdot \psi$ are linearly independent over $\mathbb{C}$ at every point of $X$. 
Proof. Let $e_{1}, J e_{1}, e_{2}, J e_{2}$ be an orthonormal frame of $T_{x} X$ with respect to the metric $h$. Write $\bar{\varepsilon}_{k}=\frac{1}{\sqrt{2}}\left(e_{k}^{*}-i\left(J e_{k}\right)^{*}\right)$ for $k=1,2$. We may assume without loss of generality

$$
a=\bar{\varepsilon}_{1} \wedge \bar{\varepsilon}_{2} \in \bigwedge^{0,2}(X, \mathbb{C}) .
$$

Note that we have $\omega_{x}=e_{1}^{*} \wedge\left(J e_{1}\right)^{*}+e_{2}^{*} \wedge\left(J e_{2}\right)^{*}$. Then a direct calculation shows that $\omega_{x} \cdot a=2 i a$, where '.' denotes the Clifford multiplication within the Clifford algebra with respect to the metric $h$.

On the other hand, we have $S(\omega)=\frac{4}{|\psi|^{2}} \omega$ : First of all, since $S(\omega)$ is a parallel 2form, we have that $S(\omega) \cdot \psi$ is a parallel field of $S_{\mathbb{C}}^{+}\left(\tilde{P}_{g}\right)$. Therefore, $S(\omega) \cdot \psi=z \psi$ for some complex number $z$ by the condition that the vector space of parallel positive spin fields is of dimension 1 . Since $S(\omega)$ is a real-valued self-dual 2 -form, it should act as a skew adjoint traceless endomorphism. Thus $z$ must be a purely imaginary number, say, $-r i$ for some real number $r$. Note that both $\omega, S(\omega)$ act as traceless skew adjoint endomorphisms and that $\omega \cdot \psi=-i \frac{|\psi|^{2}}{2} \psi$. Thus we conclude that $S(\omega)=\frac{2 r}{\psi^{2}} \omega$. In fact, since $S$ is positive definite, $r$ must be a positive real number. Furthermore we note that $S(\omega) \cdot S(\omega)=S(\omega \cdot \omega)=S\left(2 \Omega_{h}-2\right)=2 \Omega_{g}-2$, which acts as just the multiplication by -4 on $S_{\mathbb{C}}^{+}\left(\tilde{P}_{g}\right)$. Thus we have $r=2$.

Note that $S\left(\omega_{x}\right) \cdot\left(S(a) \cdot \psi_{x}\right)=S(\omega \cdot a) \psi_{x}=2 i S(a) \cdot \psi_{x}$ and $S\left(\omega_{x}\right) \cdot \psi_{x}=$ $\frac{4}{\left\lfloor\left.\right|^{2}\right.} \omega \cdot \psi_{x}=-2 i \psi_{x}$. Thus $S(a) \cdot \psi_{x}, \psi_{x}$ are respectively in eigenspaces of $S(\omega)$ with eigenvalues $\pm 2 i$. Thus it is enough to show that $S(a) \cdot \psi_{x} \neq 0$.

Let $\psi_{x}^{c} \in S_{\mathbb{C}}^{+}\left(\tilde{P}_{g}\right)_{x}$ be such that $\left\langle\psi_{x}, \psi_{x}^{c}\right\rangle=0, \psi_{x}^{c} \neq 0$. Note that $\psi_{x}^{c}$ is an eigenvector with eigenvalue $2 i$ of $S\left(\omega_{x}\right)=\frac{4}{|\psi|^{2}} \omega$. Then since $S\left(\omega_{x}\right) \cdot\left(S(a) \cdot \psi_{x}^{c}\right)=$ $S(\omega \cdot a) \psi_{x}^{c}=2 i S(a) \cdot \psi_{x}^{c}$ as well, we must have $S(a) \cdot \psi_{x}=z_{1} \psi_{x}^{c}, S(a) \cdot \psi_{x}^{c}=z_{2} \psi_{x}^{c}$ for some complex numbers $z_{1}, z_{2}$. On the other hand a direct calculation shows that $a \cdot a=0$. Since $S(a) \in \operatorname{End}_{\mathbb{C}}\left(S_{\mathbb{C}}^{+}\left(\tilde{P}_{g}\right)\right)_{x}$ is not the zero homomorphism, we must have $z_{1} \neq 0, z_{2}=0$. Thus $S(a) \cdot \psi_{x} \neq 0$. This completes the proof of Case 1 .

Case 2: The vector space of parallel positive spin fields is of rank 2. Considering Lemma 4.1, it is enough to show that $S_{\mathbb{C}}^{+}\left(\tilde{P}_{\omega, J}\right) \equiv \bigwedge^{0, \text { even }}(X, \mathbb{C})$ is a trivial complex bundle.

Let $\psi_{1}, \psi_{2}$ be parallel positive spinors in $S_{\mathbb{C}}^{+}\left(\tilde{P}_{g}\right)$ which are orthonormal with respect to the Hermitian metric. Then the endomorphisms of $S_{\mathbb{C}}^{+}\left(\tilde{P}_{g}\right), q\left(\sqrt{2} \psi_{1}\right)$, $q\left(\psi_{1}+\psi_{2}\right)$ and $i q\left(\sqrt{2} \psi_{1}\right) \circ q\left(\psi_{1}+\psi_{2}\right)$, where 'o' means the composition, are represented by the matrices

$$
\left(\begin{array}{cc}
1 & 0 \\
0 & -1
\end{array}\right),\left(\begin{array}{ll}
0 & 1 \\
1 & 0
\end{array}\right),\left(\begin{array}{cc}
0 & i \\
-i & 0
\end{array}\right)
$$

with respect to the frame field $\psi_{1}, \psi_{2}$. These are self-adjoint traceless endomorphisms of $S_{\mathbb{C}}^{+}\left(\tilde{P}_{g}\right)$ and therefore corresponds to self-dual purely imaginary two forms, say, $\omega_{1}, \omega_{2}$ and $\omega_{3}$, which form a parallel frame field for $\bigwedge_{+, g}^{2}(X ; i \mathbb{R})$.

On the other hand, there is the isomorphism $S^{-1}:\left(T^{*} X, g\right) \rightarrow\left(T^{*} X, h\right)$ between Riemannian vector bundles (see the 2nd paragraph of Case 1 above), which induces an isomorphism $S^{-1}: \bigwedge_{+, g}^{2}(X ; i \mathbb{R}) \rightarrow \bigwedge_{+, h}^{2}(X ; i \mathbb{R})$. Parallel sections are preserved by this map. Thus, $S^{-1}\left(\omega_{1}\right), S^{-1}\left(\omega_{2}\right)$ and $S^{-1}\left(\omega_{3}\right)$ form a parallel frame for $\bigwedge_{+, h}^{2}(X ; i \mathbb{R})$. 
Now note that the canonical $\operatorname{spin}^{c}$ structure of the Kähler structure $(\omega, J)$ admits a non-zero positive spinor $\psi_{0}$, that is, the non-zero constant function which is understood as a section of $\bigwedge^{0, \text { even }}(X, \mathbb{C}) \equiv S_{\mathbb{C}}^{+}\left(\tilde{P}_{\omega, J}\right)$.

By letting $S^{-1}\left(\omega_{1}\right), S^{-1}\left(\omega_{2}\right)$ and $S^{-1}\left(\omega_{3}\right)$ act on $\psi_{0}$, we have three parallel positive spin fields which are linearly independent over $\mathbb{R}$. It follows that the complex positive spin bundle $S_{\mathbb{C}}^{+}\left(\tilde{P}_{\omega, J}\right)$, which is of rank 2 , has a parallel frame field and hence it is a trivial complex vector bundle.

\section{REFERENCES}

[1] N. Hitchin, Harmonic spinors, Adv. in Math. 14 (1974), 1-55. MR 50:11332

[2] J. W. Morgan, The Seiberg-Witten equations and applications to the theory of smooth fourmanifolds, Princeton University Press, 1996. MR 97d:57042

[3] A. Moroianu, Parallel and Killing spinors on spin ${ }^{c}$ manifolds, Comm. Math. Physics 187 (1997), 417-427. MR 98i:58245]

[4] D. Salamon, Spin geometry and Seiberg-Witten invariants, 1995.

[5] D. Salamon and D. McDuff, Introduction to Symplectic Topology, Oxford University Press, 1995. MR 97b:58062

[6] C. H. Taubes, The Seiberg-Witten invariants and symplectic forms, Math. Research Letters 1 (1994) 809-822. MR 95j:57039

[7] M. Y. Wang, Parallel spinors and parallel forms, Ann. Global Anal. Geom. 7 (1989) 59-68. MR 91g:53053

Department of Mathematics, College of Natural Science, Hanyang University, Sungdong-Gu, Seoul 133-791, Korea

E-mail address: yhbyun@fermat.hanyang.ac.kr

Department of Mathematics, College of Natural Science, Inha University, IncheonSI 402-751, KoreA

E-mail address: ywlee@math.inha.ac.kr

Department of Mathematics, College of Natural Science, Dongguk University, JoOnG-Gu, SeOul 100-715, Korea

E-mail address: jpark@cakra.dongguk.ac.kr

Department of Mathematics Education, College of Education, Hongik University, Mapo-Gu, Seoul 121-791, Korea

E-mail address: jsryu@math.hongik.ac.kr 